\title{
SYSTEMS THEORY OF SYSTEMS THINKING: GENERAL AND PARTICULAR WITHIN MODERN SCIENCE AND TECHNOLOGY EDUCATION
}

\author{
Andris Broks \\ University of Latvia, Latvia
}

Following the concept of human as spiritual alive body, human thinking is spiritual activity of our brain brainwork. Today we accept that thinking means processing of information what is important for management of human body life. Studying organization of human brainwork, people have discovered that all our thoughts and thinking activities are interconnected and form definite hierarchic structures. Humans reflect world as a set or network of diverse phenomena by building corresponding theories or models of observed phenomena. In other words, all phenomena in human world of thoughts are reflected as SYSTEMS, which are organizational units of Systems Thinking. Every system contains their interconnected parts and as a whole is a part of surrounding medium, made from other systems. Such hierarchic integral understanding and comprehension of human brainwork today has become the basic value of Systems Theory as theory of Systems Thinking.

Terms "system", "systems approach" already are widely used in our professional practice and represent many particular applied systems theories. At the same time, it has become clear that there is a fundamental set of some general concepts and principles, forming core of all particular systems theories. Today it seems to be purposeful to separate general and particular within systems theory and talk about compact and exhaustive general systems theory and particular applied systems theories. To be shorter, we can introduce term "systemology", what means applied systems theory within definite branch of our life activities. For example, "Systemology of Education", "Systemology of Decision Making", "Systemology of Scientific Research" etc.

Following the core principle of overall interconnection within systems thinking, philosophical terms "general" and "particular" today we need closer connections with psychology of thinking. It is especially important for the development of our Educational Systems, balancing general and particular/professional education as well as scientific, artistic and pragmatic educational programs and corresponding particular subjects or courses. In other words, when studying hierarchic structures of thoughts as well as thinking activities, it seems that now there is an actual need for Systems Thinking as a general theory of Systems Thinking above applied system theories, what are developed for concrete branches of practical systems thinking.

Today it is very important not to lose orientation within our practical life because of global explosion of information. Professional training has to develop mastery of practitioners at much higher theoretical level of thinking than before, because simple professions are becoming computerized and virtual robots are pushing out of employment corresponding people - bio robots. Today we do not need so many professional solders, clerks etc. like simple servants, who are trained only to push buttons and ugly enjoy consumer's life. 21-st century needs constructivist approach based balanced general and professional education what means realization of optimal interconnections between particular/applied and general Systems Thinking. Alarming development of unemployment within our young people generation is partly connected with their pure general education by means of low level of Systems Thinking. What education for what life - at first we need to help our young people to develop their Systems Thinking for orientation in our modern life. Origin of human Systems Thinking is given to all of us by Mother Nature, but during our life we need further development of this natural background. It is fundamental task of our educational activities and let us notice that it is not enough to advocate only critical thinking. 


\section{Cognition}

Consideration

Behaviour

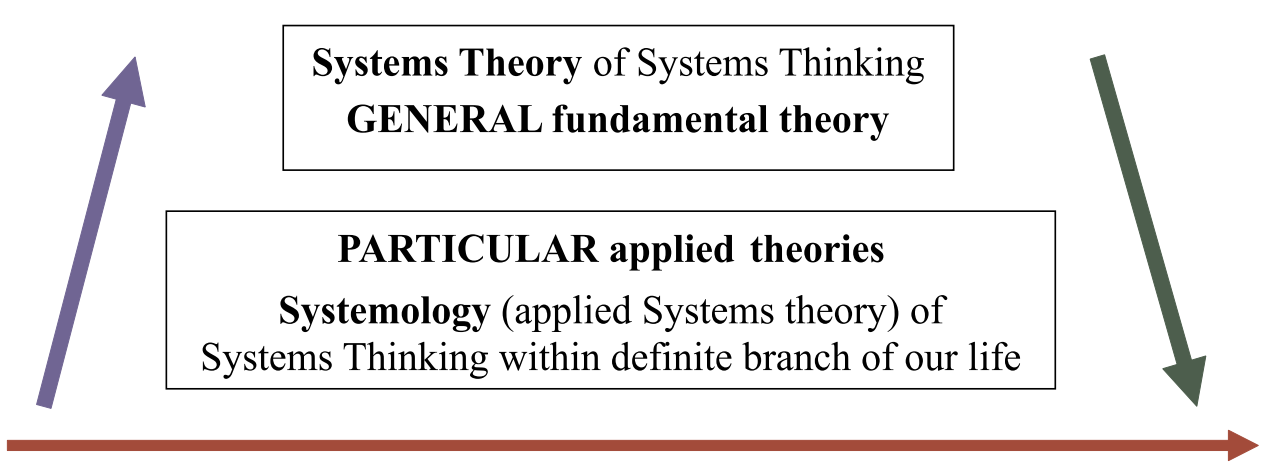

It is worth to note, that in practice we meet different Systems Thinking visualizations. Traditional basic coding of thoughts by written words and word structures are supplemented with other corresponding signs, many of them are well known from mathematics, engineering, traffic organization etc. Similar to geographic maps what are used for schematic description of interconnected objects in space, thought structures can be presented as so-called mind maps. Such schematic presentation of thought structures is widely used in modern digital communication technologies. Well-produced schemas with some comments provide rational, short and clear visualization of interconnections without using long written texts.

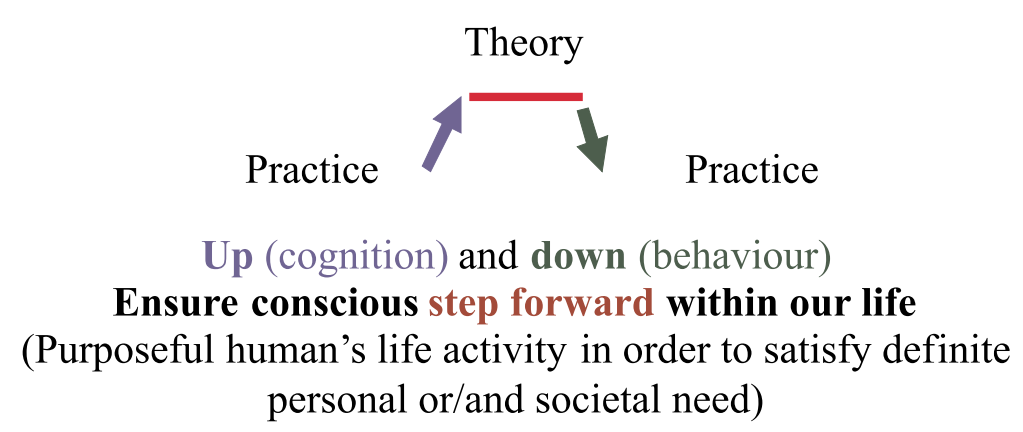

Systems Theory as general theory of Systems Thinking is just a great tool for solving many problems of 21-st century - century of fascinating development of modern Information Technologies. Perpetual changes all around us and within us, sustainable development of our individual / personal and collective / societal life needs purposeful systemic management, high quality Systems Thinking at least for our life leaders.

Finally, there are just few fundamental verities of Systems Theory.

- Human is reflecting world phenomena in human's world of thoughts as systems.

- System is a totality of systems interconnected parts and as a whole, each system is a part of its surrounding medium, made from other systems.

- People are interested to study and use systems properties to satisfy their corresponding needs of life.

- Human cognizes world by parts, comparing these parts and joining them together. Analysis, comparing and synthesis are three fundamental operations of systems thinking.

- Cause of everything (of diverse phenomena - bodies, changes of bodies, properties of bodies and its changes) is interconnection of everything. 
Good luck to all of you who are solving modern problems of sustainable development at global as well as also at local levels. General and particular, global and local solutions are systemic - they are interconnected and Systems Theory of Systems Thinking can help to get general understanding and comprehension of these interconnections and avoid many possible failures.

Our JBSE always is ready to accept and disseminate your systemic scientific research findings! Systems thinking will help you to reach high quality standards of your work and publications!

Received: July 15, 2016

Accepted: August 25, 2016 